\title{
ARTíCULO \\ Biología y pesquería del camarón Litopenaeus schmitti en la Laguna de Tacarigua, Venezuela
}

\author{
Biology and fishery of shrimp Litopenaeus schmitti in the Laguna de Tacarigua, Venezuela
}

\begin{abstract}
Juan Gassman ${ }^{1}$ y Héctor López Rojas ${ }^{2}$
'Postgrado en Zoología, Instituto de Zoología y Ecología Tropical, Universidad Central de Venezuela, Caracas 1040, Venezuela. gassman.j@gmail.com

${ }^{2}$ Laboratorio de Ictiología, Instituto de Zoología y Ecología Tropical, Universidad Central de Venezuela, Caracas 1040, Venezuela

Abstract.- In the coastal lagoon Laguna de Tacarigua there is an important fishing activity focused on shrimp and other fishes; however, very few fisheries evaluations on its resources have been conducted. We discuss aspects of reproduction, growth, mortality, recruitment and fishing of Litopenaeus schmitti in this zone. Sampling of artisanal fisheries were conducted from July 2009 to September 2010, where 3927 individuals were examined. The sex ratio showed significant differences (1:1.48) and females were more abundant. Length at maturity $\left(\mathrm{Lm}_{50}\right)$ was 13 and $13.5 \mathrm{~cm}$ for males and females, respectively. Spawning of the species was continuous throughout the year, with higher intensity between December and April. The estimated growth parameters of von Bertalanffy model were expressed as: $L t=17.5\left(1-\mathrm{e}^{-1.66(t-0.016)}\right)$ for females and $L t=14.8\left(1-\mathrm{e}^{-1.56(t-0.039)}\right)$ for males. The relative abundance (CPUE) varied throughout the year, with maximum value in December $\left(4.40 \mathrm{~kg} \mathrm{~h}^{-1}\right)$. Mortality values $\left(Z=5.49\right.$ year $^{-1}$ and $M=1.63$ to 2.99 year $^{-1}$ ) and the exploitation rate suggest that the resource is likely in a state of overexploitation, so it is necessary to establish management measures aimed at reducing fishing effort and to increase the catch length of $L$. schmitti.
\end{abstract}

Key words: Litopenaeus schmitti, reproduction, growth, mortality, shrimp

Resumen.- En la Laguna de Tacarigua se efectúa una importante actividad de pesca artesanal dirigida a la captura del camarón y otros peces; sin embargo, son escasas las evaluaciones que sobre sus recursos pesqueros se han realizado. En el presente trabajo se analizan por primera vez aspectos de la reproducción, crecimiento, mortalidad, reclutamiento y pesca de Litopenaeus schmitti en la Laguna de Tacarigua. Se efectuaron muestreos de la pesca artesanal de julio 2009 a septiembre 2010, en donde se examinaron un total de 3927 individuos. La proporción sexual mostró diferencias significativas siendo las hembras más numerosas $(1: 1,48)$. Se estimó una talla de madurez sexual $\left(\operatorname{Lm}_{50}\right)$ de 13 y $13,5 \mathrm{~cm}$ para machos y hembras, respectivamente. Se evidenció actividad reproductiva durante todo el año, con una mayor intensidad entre diciembre y abril. Los parámetros de crecimiento estimados del modelo de von Bertalanffy quedaron expresados como: $\mathrm{Lt}=17,5\left(1-\mathrm{e}^{-1,66(t-0,016)}\right)$ para hembras y $\mathrm{Lt}=14,8\left(1-\mathrm{e}^{-1,56(t-0,039)}\right)$ para machos. La abundancia relativa (CPUE) varió a lo largo de todo el año, con un valor máximo en diciembre $\left(4,40 \mathrm{~kg} \mathrm{~h}^{-1}\right)$. Los valores de mortalidad $\left(Z=5,49\right.$ año $0^{-1}$ y $M=1,63$ a 2,99 año-1) determinaron una tasa de explotación que sugiere que el recurso se encuentra en un probable estado de sobreexplotación, por lo cual es necesario establecer medidas de manejo tendientes a disminuir el esfuerzo de pesca e incrementar la talla de captura del recurso.

Palabras clave: Litopenaeus schmitti, reproducción, crecimiento, mortalidad, camarón

\section{INTRODUCCIÓN}

El camarón blanco Litopenaeus schmitti (Burkenroad, 1936), con una distribución en zonas intertropicales y subtropicales del océano Atlántico occidental que se extiende desde el sur de los Estados Unidos (o golfo de México) hasta el sur de Brasil, es una especie que habita zonas costeras generalmente influidas por deltas y estuarios de fondos areno-fangosos ricos en materia orgánica. Posee un corto y complejo ciclo de vida que involucra una fase juvenil asociada a estuarios o lagunas costeras y una fase adulta en aguas marinas a las que migra para reproducirse (Pérez-Farfante 1970, García \& Le Reste 1986). L. schmitti es el camarón peneido de mayor importancia comercial en Venezuela, aprovechado de forma artesanal en diferentes lagunas costeras y se captura desde el Lago de Maracaibo y Golfo de Venezuela, hasta el delta del río Orinoco (Novoa et al. 1998).

Uno de estos ecosistemas altamente dinámicos, propicios para la captura de $L$. schmitti, es la Laguna de Tacarigua, con características ambientales y fisicoquímicas muy particulares, especialmente por su estrecha relación temporal o permanente con el mar, que le confiere una alta diversidad de especies, heterogeneidad de hábitat y alta productividad (Ramírez 1996, Gassman \& López 2015). Este tipo de ambientes son reconocidos como importantes sitios de cría y crecimiento de las fases juveniles de numerosas especies de importancia 
comercial (Ramos-Cruz 2000, Cervigón 2005). En la Laguna de Tacarigua, Parque Nacional y Sitio Ramsar desde 1996 por su importancia como reservorio de biodiversidad, se lleva a cabo una importante actividad de pesca artesanal de carácter multiespecífica, basada en la extracción de peces y crustáceos, en donde L. schmitti es uno de los principales recursos comerciales debido a su significativo valor económico. A pesar de esto, actualmente en esta zona no existen controles ni medidas de manejo para su captura.

La opinión más generalizada es que casi todas las especies de camarones están alta o completamente explotadas, e incluso algunas sobreexplotadas y que el conocimiento necesario para proteger y conservar estos recursos es escaso (García \& Le Reste 1986, FAO 2014). En Venezuela, las mayores capturas de la especie provenían de la pesca industrial de arrastre; sin embargo, con la prohibición de esta actividad en 2008, se generó un incremento de las actividades de pesca de arrastre artesanal en zonas costeras, la cual no está regulada y generalmente es considerada ilegal, lo que ha generado un aumento en la presión de pesca sobre el recurso en estas áreas. Por ello, el presente trabajo tiene como objetivo evaluar el estado de explotación de L. schmitti en la Laguna de Tacarigua y proveer información de la pesca, reproducción, crecimiento, mortalidad y reclutamiento de la especie, que pueda contribuir al establecimiento de medidas de manejo para su aprovechamiento responsable.

\section{Materiales Y MÉTOdOS}

\section{Área de ESTUdio}

La Laguna de Tacarigua, es una laguna costera ubicada al sur del mar Caribe en la región central de la costa venezolana (10¹1'30”-10²0’20”N; 6541'10”-6557’20”O) (Fig. 1), con aproximadamente 7800 ha y una profundidad promedio de 1,2 m. La barra litoral que la separa del mar comprende $28,8 \mathrm{~km}$ de largo y el ancho oscila entre los 300 y los $1000 \mathrm{~m}$ (Novo et al. 1997). El área presenta un clima semiárido, con una precipitación máxima anual de $990 \mathrm{~mm}$. La temperatura media anual es de $26,5^{\circ} \mathrm{C}$, con una evaporación media anual de 2004 mm. La dirección de vientos es este-noreste (Alisios) con una velocidad media anual de $22,7 \mathrm{~km} \mathrm{~h}^{-1}$. La marea es diurna con una amplitud máxima de 0,30 m (Ramírez 1996).

Se llevaron a cabo muestreos mensuales a faenas de pesca artesanal de julio 2009 a septiembre 2010. La boca de la laguna se mantuvo cerrada ( $\sin$ comunicación con el mar) durante 9 meses (septiembre 2009 a mayo 2010). Se examinaron ejemplares de L. schmitti registrando su longitud total (L), desde el extremo distal de la espina rostral hasta la punta del telson $(\mathrm{cm})$ y el peso total $(\mathrm{P})(\mathrm{g})$. El sexo y la madurez sexual de los individuos se determinaron de acuerdo a la presencia de caracteres morfológicos externos como el télico en las hembras y el petasma en los machos, así como el examen macroscópico de las gónadas según lo señalado por Remoissenet (1988), Artiles et al. (1999), Pérez (2005) y Ramos-Cruz (2009).

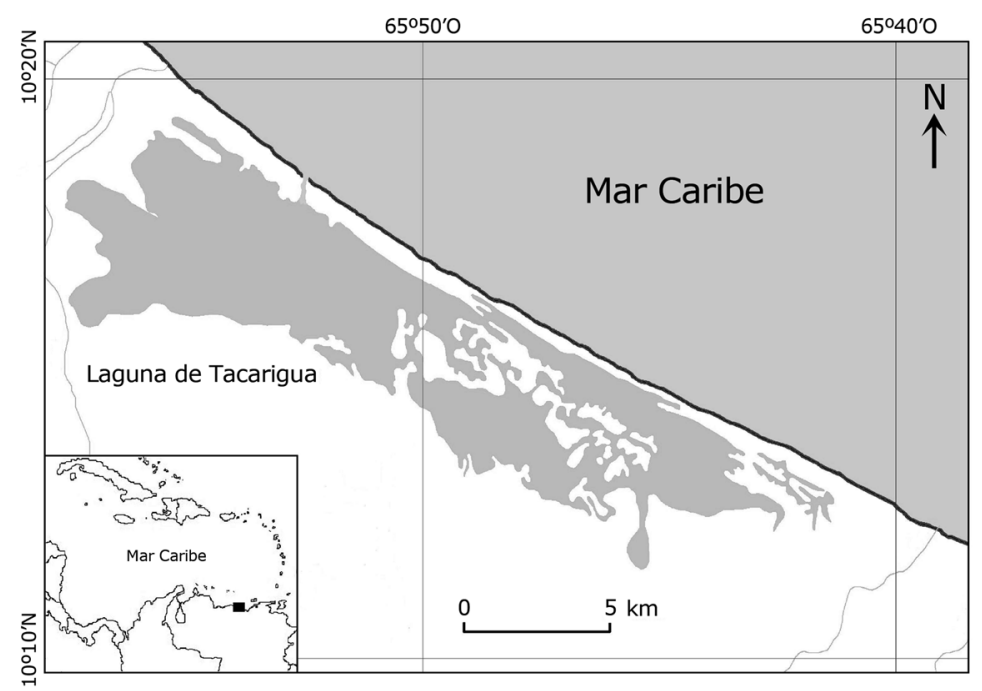

Figura 1. Ubicación geográfica del área de estudio / Geographical localization of the study area 
Para determinar la existencia de diferencias significativas en la proporción sexual mensual y total se aplicó la prueba Chicuadrado $\left(\chi^{2}, P<0,05\right)$ (Zar 1996). La talla media de madurez sexual $\left(\mathrm{Lm}_{50}\right)$ fue estimada con la fracción de ejemplares maduros en cada intervalo de talla, mediante la ecuación de la curva logística Hoydal et al. (1982):

$$
\mathrm{FM}_{(\mathrm{L})}=1 / 1+\mathrm{e}^{(\mathrm{S} 1-\mathrm{S} 2 * \mathrm{~L})}
$$

donde $\mathrm{FM}_{(\mathrm{L})}$ es la fracción de madurez a cada talla, L es la marca de clase, $S_{1}$ y $S_{2}$ se calcularon con la ecuación de la regresión lineal:

$$
\operatorname{Ln}\left(1 / \mathrm{FM}_{(\mathrm{L})}-1\right)=\mathrm{S}_{1}-\mathrm{S}_{2} * \mathrm{~L}
$$

donde $\mathrm{F}_{(\mathrm{L})}$ es la fracción a cada talla, L es la marca de Clase, $\mathrm{S}_{1}=$ a y $\mathrm{S}_{2}=-\mathrm{b}$ (Gaertner \& Laloé 1986).

La relación longitud-peso se obtuvo mediante la ecuación de Ricker (1975):

$$
\mathrm{P}=\mathrm{a} * \mathrm{~L}^{\mathrm{b}}
$$

donde a es el intercepto del eje de las ordenadas y b es la pendiente de la curva. Se realizó una prueba t-student para determinar la existencia de diferencia significativa entre sexos (Zar 1996). Los parámetros de crecimiento se estimaron mediante el análisis de las distribuciones mensuales de las frecuencias de tallas. La longitud asintótica $\left(\mathrm{L}_{\infty}\right)$ se estimó mediante el método de Powell (1979) y Wetherall (1986), el coeficiente de crecimiento (k) y una segunda estimación de $\mathrm{L}_{\infty}$, a través de la rutina ELEFAN I del programa FISAT II y se tomaron en consideración los valores más altos de la bondad de ajuste (Rn) (Gayanilo et al. 2005). El t se calculó mediante la ecuación de Pauly (1979):

$$
\log \left(-\mathrm{t}_{0}\right)=-0,3922-0,2752 * \log \mathrm{L}_{\infty}-1,038 * \log \mathrm{k}
$$

para seguidamente estimar las curvas de crecimiento para machos y hembras ajustadas al modelo de von Bertalanffy (1948):

$$
\mathrm{Lt}=\mathrm{L}_{\infty}\left(1-\mathrm{e}^{-\mathrm{k}(\mathrm{t}-\mathrm{t} 0)}\right)
$$

donde $\mathrm{L}(\mathrm{t})$ es la longitud a la edad $\mathrm{t}$, $\mathrm{t}$ la edad del pez $\mathrm{y} \mathrm{t}_{0}$ la edad hipotética a la cual el individuo tiene la longitud 0 . El índice de crecimiento phi prima ( $\varnothing^{\prime}$ ) se estimó mediante la ecuación de Pauly \& Munro (1984):

$$
\varnothing^{\prime}=\log 10 \mathrm{k}+2 \log 10 \mathrm{~L}_{\infty}
$$

Se estimó la mortalidad total (Z) por el método de la curva de captura convertida a longitudes de Pauly et al. (1995):

$$
\operatorname{Ln}(\mathrm{Ni} / \Delta \mathrm{ti})=\mathrm{a}+\mathrm{bti}
$$

donde Ni es el número de individuos capturados en un intervalo de clase $\mathrm{i}$, a y b son estimados a través de análisis de regresión realizada con los puntos de la fase explotada en la curva de captura, la pendiente de $\mathrm{b}$ con el signo cambiado es la $\mathrm{Z}$ estimada, ti es la edad relativa del intervalo de clase i y $\Delta$ ti es el tiempo requerido por el individuo para su crecimiento a través de la clase i. La mortalidad natural (M) se calculó mediante la ecuación empírica de Pauly (1984):

$\log \mathrm{M}=0,0066-0,279 * \log \mathrm{L}_{\infty}+0,6543 * \log \mathrm{k}+0,4632 * \log \mathrm{T}(8)$

donde $\mathrm{T}$ es la temperatura media anual $\left(30,5^{\circ} \mathrm{C}\right.$ en este caso) y una segunda estimación por el método de Rikhter \& Efanov (1976):

$$
\mathrm{M}=\left(1,52 /\left(\operatorname{Tm}_{50 \%}{ }^{0,72}\right)-0,16\right.
$$

donde $\mathrm{Tm}_{50 \%}$ es la edad de maduración masiva, la cual se estimó introduciendo la talla de madurez $\left(\mathrm{Lm}_{50}\right)$ en la ecuación inversa de von Bertalanffy (Sparre \& Venema 1997):

$$
\mathrm{t}\left(\mathrm{L}_{\mathrm{L}}\right)=\mathrm{t}_{0}-(1 / \mathrm{k}) * \ln \left(1-\left(\mathrm{Lm}_{50} / \mathrm{L}_{\infty}\right)\right.
$$

La mortalidad por pesca (F) y la tasa de explotación (E) se calcularon con las fórmulas:

$$
\begin{aligned}
& F=Z-M \\
& y \\
& E=F / Z
\end{aligned}
$$

El patrón de reclutamiento se obtuvo mediante la proyección de los datos de frecuencia de tallas reconstruidos en el tiempo con los parámetros de crecimiento estimados en escala de un año (Gayanilo et al. 2005).

La Captura por Unidad de Esfuerzo (CPUE), como índice de abundancia relativa, fue definida como kilogramos capturados por horas de pesca $\left(\mathrm{kg} \mathrm{h}^{-1}\right)$. Para determinar diferencias significativas en la CPUE mensual se realizó un ANDEVA de una vía y posteriormente se usó el test de Tukey para determinar diferencias entre las medias de la CPUE mensual.

\section{Resultados}

La pesca en la Laguna de Tacarigua es de tipo artesanal de pequeña escala, realizada por pescadores a volapié o con la utilización de embarcaciones tipo canoa de fibra de vidrio o madera de entre 4,6 y 6 m de eslora, impulsadas en su mayoría por palanca o motores fuera de borda de baja potencia (hasta $15 \mathrm{HP}$ ). La captura de camarón es realizada con atarraya camaronera (en promedio 3,66 $\mathrm{m}$ de largo y 2,60 $\mathrm{m}$ de alto con una abertura de malla de $1,5 \mathrm{~cm}$ ), generalmente en horarios nocturnos, aprovechando la migración diaria del camarón hacia la salida al mar. Las faenas se realizan principalmente en grupos de dos pescadores por embarcación y duran entre 4 y 6 horas 
diarias. Los reportes de las capturas anuales de camarón en la zona presentan importantes fluctuaciones anuales (2008: 4,6 t, 2009: 2,5 t, 2010: 5,8 y 2011: 13,6 t).

Se registraron 101 faenas de pesca de L. schmitti y se examinaron un total de 3927 ejemplares con tallas comprendidas entre 3,7 y $17 \mathrm{~cm}$ de L (Fig. 2), con una talla promedio para ambos sexos de $9,93 \mathrm{~cm}$ y una moda de 8,6 $\mathrm{cm}$. La L de las hembras varió entre 5 y $17 \mathrm{~cm}$, mientras que para los machos entre 4,6 y $15,5 \mathrm{~cm}$.

\section{REPRODUCCIÓN}

Del total de individuos 2000 fueron hembras (50,9\%), 1355 machos $(34,5 \%)$ y 572 de sexo indeterminado $(14,6 \%)$. La proporción sexual mostró diferencias significativas para la totalidad del periodo de estudio, siendo las hembras más numerosas $1: 1,48\left(\chi^{2}=124\right.$, g.l. $\left.=1, P<0,05\right)$, aunque no se encontraron diferencias en agosto $\left(\chi^{2}=3,53\right.$, g.l. $\left.=1, P>0,05\right)$, enero $\left(\chi^{2}=0,11\right.$, g.l. $\left.=1, P>0,05\right)$, febrero $\left(\chi^{2}=0,31\right.$, g.l. $=1$, $P>0,05)$ y marzo $\left(\chi^{2}=2,77\right.$, g.l. $\left.=1, P>0,05\right)$. Individuos maduros fueron observados durante todo el año, excepto en mayo, junio y julio, cuando la especie desapareció de las capturas en la laguna. Sin embargo, se observó un periodo de mayor presencia de individuos maduros entre diciembre y abril (Fig. 3). La Lm $_{50}$ fue estimada en 13,5 para hembras $\left(\mathrm{S}_{1}=31,25\right.$ y $\left.\mathrm{S}_{2}=2,31\right)$ y $13 \mathrm{~cm}$ para machos $\left(\mathrm{S}_{1}=24,48\right.$ y $\left.\mathrm{S}_{2}=1,88\right)$ (Fig. 4).

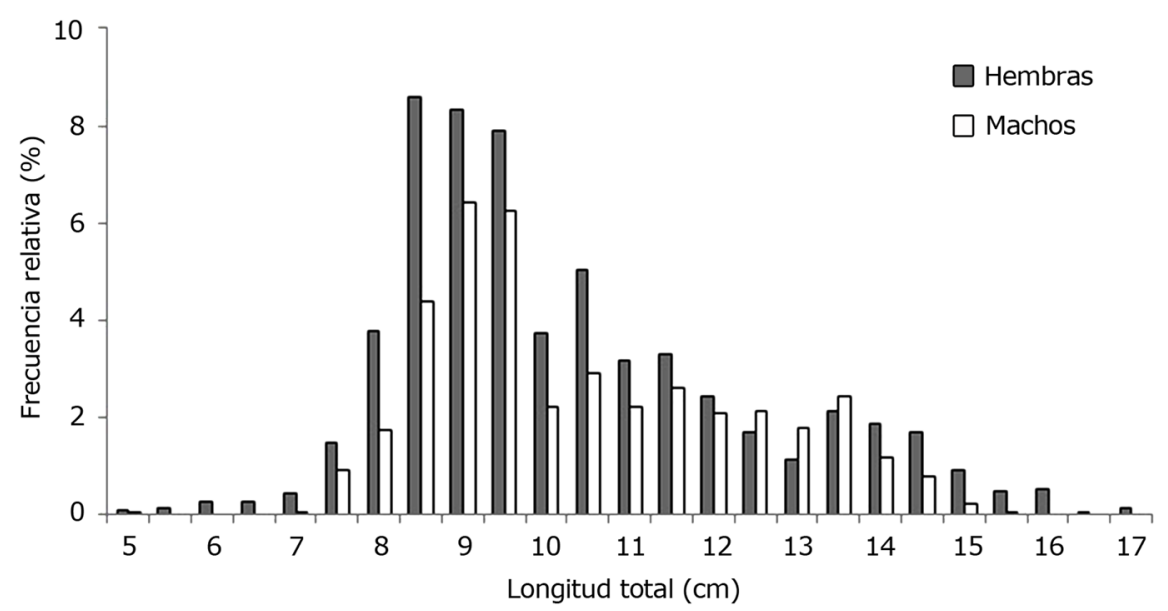

Figura 2. Distribución de frecuencia de tallas de Litopenaeus schmitti en la Laguna de Tacarigua de julio 2009 a septiembre 2010 / Distribution of lengths of Litopenaeus schmitti from July 2009 to September 2010 in the Laguna de Tacarigua, Venezuela

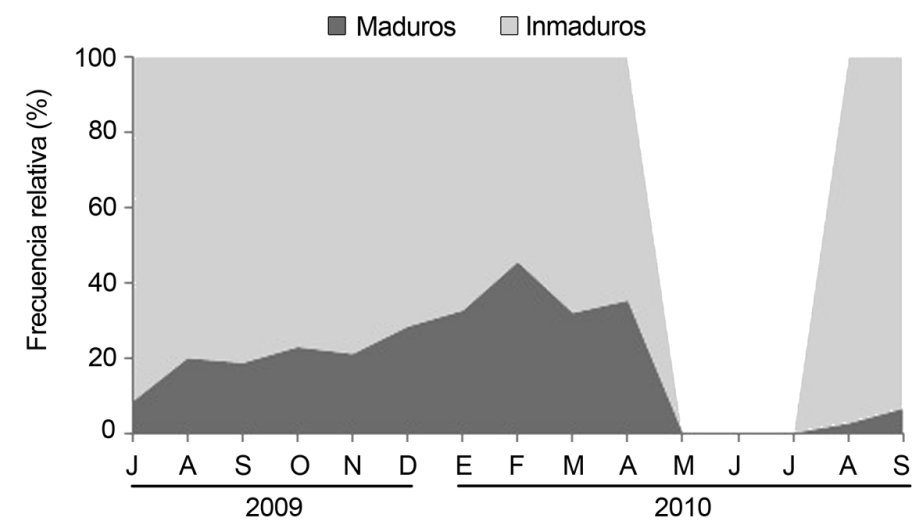

Figura 3. Variación mensual de la frecuencia relativa de las fases de madurez sexual de Litopenaeus schmitti de julio 2009 a septiembre 2010 en la Laguna de Tacarigua, Venezuela / Monthly variation of relative frequency maturity stages of Litopenaeus schmitti from July 2009 to September 2010 at Laguna de Tacarigua, Venezuela 


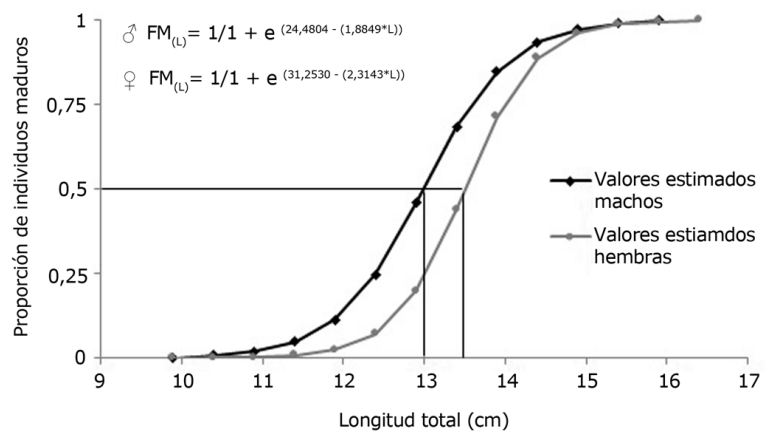

Figura 4. Talla media de madurez sexual $\left(\mathrm{Lm}_{50}\right)$ de hembras y machos de Litopenaeus schmitti en la Laguna de Tacarigua de julio 2009 a septiembre 2010 / Sexual maturity curve and size at maturity $\left(\mathrm{Lm}_{50}\right)$ of females and males of Litopenaeus schmitti at Laguna de Tacarigua, Venezuela

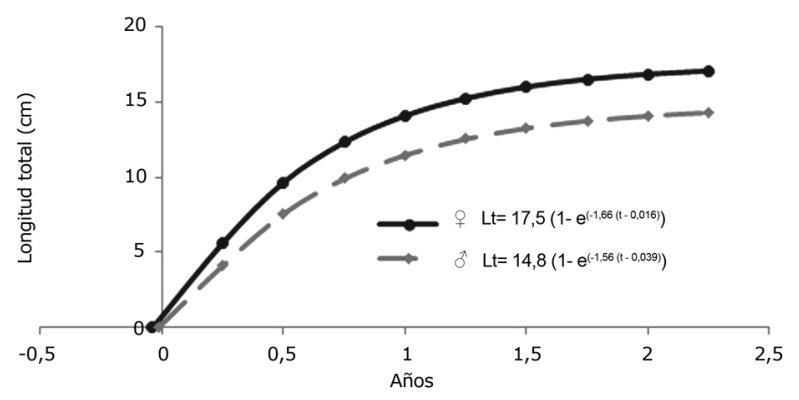

Figura 5. Curva de crecimiento ajustada al modelo de von Bertalanffy para machos y hembras de Litopenaeus schmitti en la Laguna de Tacarigua de julio 2009 a septiembre 2010 / Growth curve according to the von Bertalanffy model of females and males of Litopenaeus schmitti at Laguna de Tacarigua, Venezuela

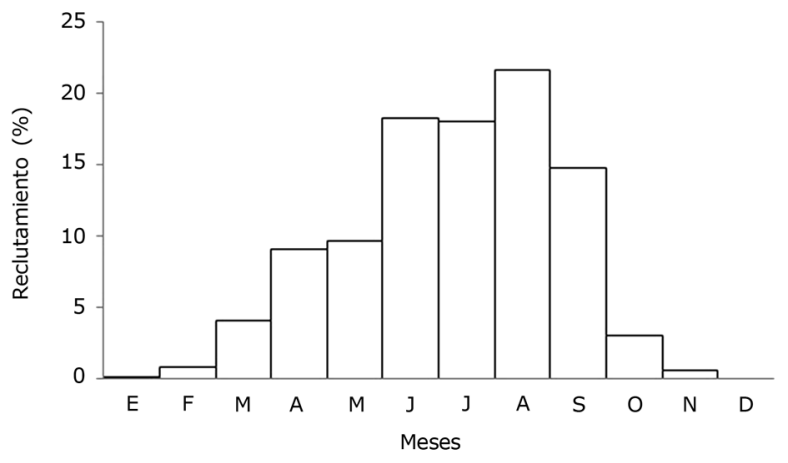

Figura 6. Patrón de reclutamiento anual de Litopenaeus schmitti estimado a partir de los parámetros de crecimiento en la Laguna de Tacarigua, Venezuela / Annual recruitment pattern for Litopenaeus schmitti estimated from growth parameters at Laguna de Tacarigua, Venezuela

\section{Crecimiento}

No se encontró diferencia significativa en el crecimiento entre machos y hembras $(t=1,61$, g.l. $=2020, P>0,05)$, quedando establecida la relación de tipo isométrico: $\mathrm{P}=0,0068 * \mathrm{~L}^{3,085}\left(\mathrm{R}^{2}=\right.$ $0,98)$. Con la rutina del ELEFAN I se definieron los valores de $\mathrm{L}_{\infty} \mathrm{y} \mathrm{k}, \mathrm{y}$ junto al valor de $\mathrm{t}_{0}$ se ajustaron al modelo de von Bertalanffy (Fig. 5) quedando expresadas las siguientes curvas: $\mathrm{Lt}=17,5\left(1-\mathrm{e}^{-1,66(\mathrm{t}-0,016)}\right)$ para hembras $(\mathrm{Rn}=162), \mathrm{Lt}=14,8(1-$ $\left.\mathrm{e}^{-1,56(\mathrm{t}-0,039)}\right)$ para machos $(\mathrm{Rn}=179)$ y $\mathrm{Lt}=17,6\left(1-\mathrm{e}^{-1,65(\mathrm{t}-0,017)}\right)$ para ambos sexos $(\mathrm{Rn}=195)$. El valor $\emptyset^{\prime}$ estimado fue de 2,71 para sexos combinados y 2,70 y 2,53 para hembras y machos, respectivamente.

\section{Mortalidad y REClutamiento}

La mortalidad total se estimó en 5,49 $\mathrm{año}^{-1}$ (con un rango de variación de 4,92 a 6,06 año ${ }^{-1}$ ). La mortalidad natural estimada con la ecuación de Pauly (1984) fue de 2,99 año ${ }^{-1}$ y por el método de Rikhter \& Efanov (1976) de 1,63 año ${ }^{-1}\left(\mathrm{Tm}_{50 \%}=\right.$ 0,796 años). La mortalidad por pesca (F) fue de 2,5 a 3,8 año ${ }^{-1}$ y una tasa de explotación (E) de 0,46 a 0,70 . Se observó un pulso máximo de reclutamiento al año de junio a septiembre (Fig. 6).

\section{Abundancia Relativa}

La CPUE varió a lo largo de todo el año, mostrando un valor máximo en diciembre $\left(4,40 \mathrm{~kg} \mathrm{~h}^{-1}\right)$ y el menor en junio y julio $\left(0,001 \mathrm{~kg} \mathrm{~h}^{-1}\right)$ (Fig. 7). El análisis estadístico confirmó diferencias significativas en los valores de la CPUE entre los meses $\left(\mathrm{F}_{(1,808)}\right.$ $=28,62, P<0,0001)$, mientras el posterior Test de Tukey determinó dos grupos donde la media de la CPUE varía temporalmente, el de mayores valores, de junio a diciembre, incluidos agosto y septiembre 2010, y el segundo de enero a julio.

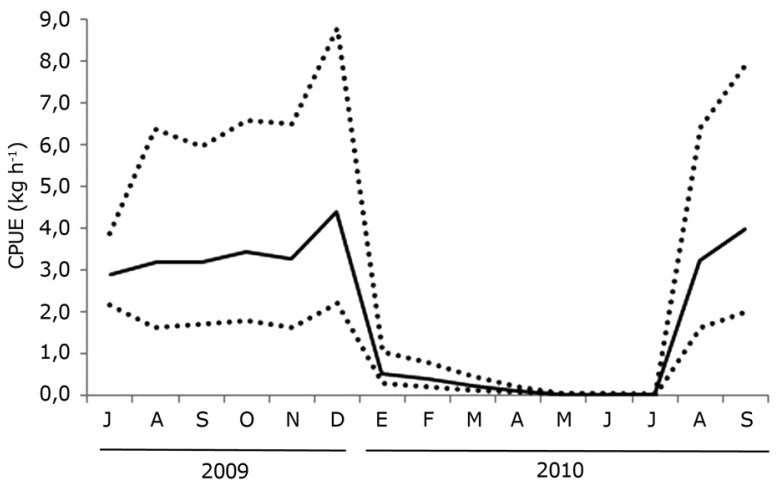

Figura 7. Variación mensual de la CPUE $\left(\mathrm{kgh}^{-1}\right)$ del camarón, Litopenaeus schmitti, en la Laguna de Tacarigua de julio 2009 a septiembre 2010 (las líneas punteadas corresponden a los intervalos de confianza al 95\%) / Monthly variation of catch per unit effort (CPUE; $\mathrm{kg} \mathrm{h}^{-1}$ ) for Litopenaeus schmitti from July 2009 to September 2010 in the Laguna de Tacarigua, Venezuela (Dotted lines represent 95\% confidence intervals) 


\section{Discusión}

Para las poblaciones aledañas a la Laguna de Tacarigua la pesca representa la principal y prácticamente única actividad económica. Esta dependencia ha ocasionado una fuerte presión sobre los recursos pesqueros que allí se extraen, haciéndolos vulnerables a la sobreexplotación, particularmente por el exceso de capacidad de pesca, lo cual es considerado como uno de los factores que más contribuye a la insostenibilidad de la pesca costera (Salas et al. 2011). En la actualidad, el numero de pescadores se ha incrementado en forma significativa, existiendo 300 aproximadamente en las décadas de los 60 y 70 (Gamboa et al. 1971) y ascendiendo hasta 600, con evidencias también de una paulatina disminución de las capturas pesqueras (Gassman 2012) y a pesar que el área posee un Plan de Ordenamiento y Reglamento de Uso (Decreto N 1643 del 1807-1991), el cual es débil e insuficiente en cuanto a las regulaciones pesqueras y particularmente para el camarón no existen ningún tipo de restricción para su captura.

El rango de tallas encontrado en las capturas de la especie en la Laguna de Tacarigua $(4,3$ a $17 \mathrm{~cm})$ fue similar a lo reportado para el Lago de Maracaibo de 7 a 17,5 cm (Andrade 2005), aunque menor a otros reportes del occidente del país con tallas de 6 a $20 \mathrm{~cm}$ (Andrade \& Stotz 1999) y de 9 a 24 $\mathrm{cm}$ en el Golfo de Venezuela (Díaz et al. 2014). Esta diferencia determinada por el ambiente lagunar del presente trabajo, considerado un área de predominancia de ejemplares juveniles y subadultos. En general las hembras alcanzaron tallas más grandes que los machos, lo cual ha sido también observado por Andrade \& Stotz (1999), que además señalan que son más numerosas en las clases de longitudes superiores.

La proporción sexual con predominancia de hembras $(1: 1,48)$ coincide con estudios previos de Andrade \& Stotz (1999) en el Lago de Maracaibo (1:1,2), Díaz et al. (2014) para el Golfo de Venezuela (1:1,5) y Dos Santos et al. (2008) para aguas del sur del Brasil (1:2,76). La presencia de individuos maduros durante todo el periodo de estudio, indica la reproducción continua a lo largo del año, tal y como es mencionado para los peneidos en general (García \& Le Reste 1986), con un periodo de mayor intensidad entre diciembre y abril. Según Altuve (1997) el proceso reproductivo de $L$. schmitti en el Golfo de Paria también tiene lugar durante todo el año, con mayor intensidad entre septiembre y noviembre. También concuerda con Dos Santos et al. (2008), quienes señalan una reproducción de junio a febrero con un pulso máximo entre noviembre y enero. Por otra parte, aunque no ha sido reportada la reproducción del camarón dentro Laguna de Tacarigua, en el mes de abril 2010 se encontró una hembra de $14,4 \mathrm{~cm}$ y 25,7 g con espermatóforo adherido, por lo que es probable que al encontrarse la boca de la laguna cerrada en ese periodo y por consiguiente limitada la migración reproductiva, algunos individuos realizaran la cópula dentro de la misma.

La $\mathrm{Lm}_{50}$ estimada en el presente trabajo coincide con lo reportado en el occidente del país de $12 \mathrm{~cm}$ para machos y 13 cm para hembras (Godoy 1971, Sangronis 1991), aunque menor a la encontrada por Altuve (1997) para el Golfo de Paria quien señala una talla mínima de madurez de $11,2 \mathrm{~cm}$ y una talla media de $16 \mathrm{~cm}$ y Dos Santos et al. (2008) quienes reportan $15,8 \mathrm{~cm}$ para hembras, lo cual puede estar asociado a la migración reproductiva de la especie. La talla a la cual los camarones inician su migración puede variar estacionalmente, como consecuencia de las condiciones tróficas y térmicas durante el crecimiento y de variaciones de la temperatura y salinidad (García \& Le Reste 1986). Sobresale que el $89 \%$ de los individuos capturados en la Laguna de Tacarigua se encontraron por debajo de la talla media de madurez.

L. schmitti entra a la pesquería artesanal probablemente a los 3 o 4 meses de edad, después del desove, con longitudes superiores a los 5 o $6 \mathrm{~cm}$ y desapareciendo, generalmente, al año y medio de vida, cuando ha alcanzado longitudes superiores a los $19 \mathrm{~cm}$ debido a su migración a aguas más profundas y alejadas de la costa (Andrade \& Stotz 1999). El reclutamiento a la pesquería en la Laguna de Tacarigua ocurrió en un periodo de mayor intensidad entre junio y septiembre, coincidiendo con Andrade \& Stotz (1999), quienes afirman que el reclutamiento ocurre durante todo el año, con 2 períodos bien definidos entre febrero a abril y de agosto a octubre, así como con Díaz et al. (2014), quienes señalan los mayores reclutamientos entre noviembre y febrero en el Golfo de Venezuela.

García \& Le Reste (1986) afirman que la distribución y abundancia de los camarones en los estuarios se caracteriza por importantes variaciones espacio-temporales, donde juegan un papel determinante los cambios en los factores ambientales. En el caso de la abundancia de L. schmitti, el efecto de la apertura y cierre de la boca de la laguna fue muy evidente. $\mathrm{Su}$ abundancia relativa presentó dos picos de mayor magnitud, el primero y más importante entre julio y diciembre (2009), seguido de una disminución progresiva a partir de enero, ya para ese entonces la laguna tenía 4 meses cerrada, por consiguiente durante ese lapso, no hubo entrada al área de nuevos reclutas y la pesquería fue capturando los individuos presentes hasta su desaparición hacia mayo 2010 y solo su abundancia se incrementa nuevamente hasta agosto 2010, justo después de la apertura de la boca, por lo cual se evidencia que la abundancia del recurso en la Laguna de Tacarigua está directamente influenciada por los aportes de reclutas de la zona marina adyacente. Andrade (2000) indica que los desembarques 
artesanales del camarón en el Lago de Maracaibo presentan marcadas fluctuaciones interanuales sin relación entre las capturas y el esfuerzo pesquero, sino que estas se deben a variaciones en la abundancia de la especie, las cuales son motivadas principalmente por parámetros ambientales. Por su parte, Díaz et al. (2013) reportan fluctuaciones en la abundancia en el Golfo de Venezuela asociadas a incrementos en el reclutamiento provenientes del Lago de Maracaibo y variaciones en la CPUE en relación a la temperatura y profundidad.

En relación al tipo de crecimiento encontrado (isométrico), los resultados difieren de lo señalado en reportes previos por Andrade \& Stotz (1999) de crecimiento alométrico negativo y Díaz et al. (2014) de alométrico positivo. Los valores de $\mathrm{L}_{\infty}$ se encuentran dentro del rango de valores reportados previamente para la especie. Sangronis (2001) para la ciénaga de Los Olivitos, ambiente de similares características al del presente estudio, reportó valores de 13,1 cm en machos y 14,2 cm en hembras. Para el Lago de Maracaibo y Golfo de Venezuela, los valores varían en machos de 17,3 a 20,1 cm y en hembras de 21 a 22,2 cm (Andrade \& Stotz 1999, Andrade \& Pérez 2007, Díaz et al. 2014), lo cual se debe a que en estas áreas marinas predominan individuos adultos, por lo cual se presentan rangos de tallas superiores para ambos sexos, mientras el presente estudio fue realizado en un área lagunar de predominancia de la fracción juvenil y subadulta, por consiguiente, la presencia de individuos mayores a 18 o $19 \mathrm{~cm}$ es menor ya que generalmente han migrado hacia las áreas marinas (Andrade 2000). En el caso de k, se evidencia un crecimiento rápido y fue superior en hembras. Los valores obtenidos son muy similares a los reportes previos: 1,2 a 1,5 año ${ }^{-1}$ (Andrade \& Stotz 1999), 1,7 a 1,9 año-1 (Sangronis 2001), 1,12 a 1,8 año $^{-1}$ (Andrade \& Pérez 2007) y 1,4 a 1,69 año $^{-1}$ (Díaz et al. 2014). De igual forma, el índice de crecimiento en el presente trabajo $(2,53$ a 2,71$)$ es consistente con los señalados previamente por los anteriores autores para la especie, los cuales varían entre 2,47 y 2,92.

La mortalidad natural en crustáceos es ampliamente variable e influida por numerosos aspectos, principalmente relacionados a variaciones estacionales en parámetros ambientales, fluctuaciones en la abundancia de predadores, variaciones poblacionales en los patrones de distribución y agregación o migraciones (Gracia 1989). En camarones peneidos, es poco lo que se conoce sobre la mortalidad, aunque se sabe que especies con un alto valor de $\mathrm{k}$, como L. schmitti, generalmente tienen una elevada mortalidad natural en las primeras fases de vida (Ramos-Cruz 2000, Andrade 2000). Los valores de mortalidad natural obtenidos en el presente trabajo $(M=2,99$ y 1,63 año ${ }^{-1}$ ) fueron similares a lo señalado en trabajos previos para la especie por Andrade (2000) de 2,04 año-1 y Díaz et al.
(2014) de 1,27 a 3,60 año-1 y pudo estar ampliamente influenciada por las variaciones en los valores de temperatura y salinidad ocasionados por el cierre de la boca de la laguna. Por el contrario, la mortalidad por pesca fue inferior a lo estimado por los autores antes citados (7,16 y de 0,36 a 4,14 año $^{-1}$, respectivamente), lo cual es de esperarse si se considera que en esa zona se encuentran las mayores flotas pesqueras asociadas al camarón y, aunque esto podría sugerir una menor presión de pesca sobre $L$. schmitti en la Laguna de Tacarigua, la tasa de explotación encontrada $(0,46-0,70)$ de acuerdo a Gulland (1971) indica que el recurso se encuentra en niveles de plena y posiblemente sobreexplotación, como ya ha sido reportado para otras regiones del país (Andrade 2000, Díaz et al. 2014).

En el caso particular de la Laguna de Tacarigua, dado el gran porcentaje de individuos inmaduros que se capturan, así como la tasa de explotación estimada, se podría estar presentando una sobrepesca por crecimiento, causada por la extracción de individuos jóvenes antes de llegar a su tamaño de madurez (Sparre \& Venema 1997), lo cual señala la necesidad de implantar controles que entre otras cosas, incrementen la talla de captura del recurso y tiendan a disminuir el esfuerzo de pesca. Por ello, entre las recomendaciones para el manejo se sugiere el establecimiento de una talla mínima de captura, el incremento de la abertura de malla del arte de pesca empleado e implementar un periodo de veda, tomando en cuenta la importancia de la interacción laguna-mar y la migración que como estrategia reproductiva posee la especie. Así mismo, se recomienda realizar estudios posteriores que involucren a la fracción de la población de camarón que se encuentra en la zona marina adyacente a la Laguna de Tacarigua, con el fin de realizar una evaluación completa del stock, necesaria para una gestión y manejo integral del recurso en el área.

\section{Agradecimientos}

A los pescadores de la Laguna de Tacarigua por su incondicional disposición. A los árbitros anónimos por sus acertadas sugerencias.

\section{LITERATURA CITADA}

Altuve D. 1997. Aspectos poblacionales y pesqueros del Camarón Blanco, Penaeus schmitti (Bunkenroad, 1936) (Crustacea: Decapoda), de la pesca artesanal en la costa norte del Golfo de Paria, Sucre, Venezuela. Tesis de Maestría, Instituto Oceanográfico de Venezuela, Universidad de Oriente, Cumaná, 138 pp.

Andrade G. 2000. Algunos aspectos biológicos y pesqueros de camarón blanco, en el Lago de Maracaibo. FONAIAP Divulga 68: 10-12. 
Andrade G. 2005. Análisis bioeconómico de la pesquería secuencial del camarón blanco, Litopenaeus schmitti, y su aplicación para el manejo del recurso en el Occidente de Venezuela. Tesis Doctoral, CINVESTAV, Unidad Mérida, Yucatán, 165 pp.

Andrade G \& E Pérez. 2007. Comparación de métodos de estimación de parámetros de crecimiento del camarón blanco Litopenaeus schmitti en el occidente de Venezuela. Interciencia 32(1): 41-47.

Andrade G \& W Stotz. 1999. Crecimiento y mortalidad del camarón blanco (Penaeus schmitti) en el Lago de Maracaibo, Venezuela. Zootecnia Tropical 17(1): 63-89.

Artiles M, E Regueira \& L Pérez. 1999. Maduración y reproducción de Penaeus schmitti utilizando hembras ablasionadas y no ablasionadas bajo iluminación natural. Revista de Investigaciones Marinas 20(1-3): 93-102.

Bertalanffy L Von. 1948. A quantitative theory of organic growth. Human Biology 10: 181-213.

Cervigón F. 2005. La ictiofauna marina de Venezuela: una aproximación ecológica. Boletín del Instituto Oceanográfico de Venezuela 44(1): 3-28.

Díaz A, O Ferrer, R Álvarez, L González, J Méndez \& M Corona. 2013. Catch per unit effort-environmental variables relations in the fishery of white shrimp (Litopenaeus schmitti) from the Gulf of Venezuela. Agricultural Sciences 4(6): 83-90.

Díaz A, O Ferrer, R Álvarez, L González, J Méndez \& M Corona. 2014. Mortality, recruitment pattern and growth of the white shrimp Litopenaeus schmitti (Crustacea: Penaeidae) from the Gulf of Venezuela. Ciencia 22(4): 187196.

Dos Santos J, E Severino \& A Dos Santos. 2008. Estrutura populacional do camarão-branco litopenaeus schmitti nas regiões estuarina e marinha da Baixada Santista, Sao Paulo, Brasil. Boletim do Instituto de Pesca 34(3): 375-389.

FAO. 2014. El estado mundial de la pesca y la acuicultura, 253 pp. FAO, Roma.

Gaertner D \& F Laloé. 1986. Etude biométrique de la taille á première maturité sexuelle de Geryon maritae Manning et Holthuis, 1981 du Sènegàl. Oceanologica Acta 9(4): 479487.

Gamboa B, A García, J Benítez \& T Okuda. 1971. Estudio de las condiciones hidrográficas y químicas en el agua de la Laguna de Tacarigua. Boletín del Instituto Oceanográfico de Venezuela 10: 55-72.

García S \& L Le Reste. 1986. Ciclos vitales, dinámica, explotación y ordenación de las poblaciones de camarones peneidos costeros. FAO Documento Técnico de Pesca 203: $1-180$.

Gassman J. 2012. Caracterización de la pesca de la Laguna de Tacarigua y algunos aspectos biológicos de las principales especies comerciales. Tesis de Maestría, Facultad de Ciencias, Universidad Central de Venezuela, Caracas, 146 pp.
Gassman J \& H López. 2015. Variación de la abundancia de los recursos pesqueros en el parque nacional Laguna de Tacarigua, Venezuela. Acta Biológica Venezuelica 35(1): 1125.

Gayanilo F, P Sparre \& D Pauly. 2005. FAO-ICLARM Stock Assessment Tools II (FiSAT II). User's guide. FAO Computerized Information Series (Fisheries) 8: 1-168.

Godoy G. 1971. Maduración y desove del camarón blanco, Penaeus schmitti Burkenroad, 1936, en el occidente de Venezuela. Tesis de Licenciatura, Facultad de Ciencias, Universidad Central de Venezuela, Caracas, 79 pp.

Gracia A. 1989. Mortalidad natural del camarón blanco Penaeus setiferus (Linnaeus, 1767) en la zona de Campeche. Anales del Instituto de Ciencias del Mar y Limnología, Universidad Nacional Autónoma de México 16: 42-53.

Gulland J. 1971. Fish resources of the ocean, 255 pp. Fishing New Books, London.

Hoydal K, C Rorvik \& P Sparre. 1982. Estimation of effective mesh sizes and their utilization in assessment. Dana 2: 6995.

Novo I, L Morales, C Rodríguez, G Martínez \& I De Hertelendy. 1997. Ciencia y conservación en el sistema de Parque Nacionales de Venezuela: una experiencia de cooperación Interinstitucional, 356 pp. Impresos Altamira, Caracas.

Novoa D, J Mendoza, L Marcano \& J Cárdenas. 1998. Atlas pesquero marítimo de Venezuela, $71 \mathrm{pp}$. MAC-SARPACONGEPESCA, Caracas.

Pauly D. 1979. Theory and management of tropical multispecies stocks: A review, with emphasis on the Southeast Asian demersal fisheries. International Center for Living Aquatic Resources Management. Studies and Reviews 1: 1-35.

Pauly D. 1984. Fish population dynamics in tropical waters: a manual for use for programmable calculators, ICLARM Studies and Reviews 8: 325.

Pauly D \& L Munro. 1984. Once more on growth comparison in fish and vertebrates. Fishbyte 2: 1-21.

Pauly D, J Moreau \& N Abad. 1995. Comparison of age structured and length-converted catch curves of brown trout Salmo trutta in two French rivers. Fisheries Research 22: 197-204.

Pérez-Farfante I. 1970. Sinopsis de datos biológicos sobre el camarón blanco Penaeus schmitti Burkenroad, 1936. FAO Fisheries Report 57: 1417-1438.

Pérez L. 2005. Fisiología y calidad reproductiva de machos de camarón blanco Litopenaeus schmitti en condiciones de cautiverio. Tesis Doctoral, Centro de Investigaciones Biológicas del Noroeste, La Paz, 132 pp.

Powell D. 1979. Estimation of mortality and growth parameters from the length frequency of a catch. Rapport process- V Réunion Conseil International pour 1'Exploitation de la Mer 175: $167-169$. 
Ramírez P. 1996. Lagunas costaneras venezolanas, 275 pp. Centro Regional de Investigaciones Ambientales, Porlamar.

Ramos-Cruz S. 2000. Composición por tallas, edad y crecimiento de Litopenaeus vannamei (Natantia: Penaeidae), en la laguna Mar Muerto, Oaxaca-Chiapas, México. Revista de Biología Tropical 48(4): 728-738.

Ramos-Cruz S. 2009. Tallas, sexo y desarrollo gonádico de Litopenaeus vannamei (Crustacea: Decapoda: Penaeidae), durante la veda de 1999 en el Golfo de Tehuantepec, México. Ciencia Pesquera 17(2): 29-39.

Remoissenet G. 1988. Consultoría en maduración de camarones peneidos (P. vannamei, $P$. stylirostris, $P$. schmitti), 38 pp. Informe Técnico FAO, Roma.< http:// www.fao.org/3/contents/7caca04d-2d22-52bb-a71e299f916e26d1/AC408S00.htm>

Ricker W. 1975. Computation and interpretation of biological statistic of fish populations. Department of environment fisheries and marine service. Bulletin Fisheries Research Board of Canada 191: 1-382.

Rikhter V \& V Efanov. 1976. On one of the approaches to estimation of natural mortality of fish populations. ICNAF Res. Doc. 76/VI/8, 12 pp.
Salas S, R Chuenpagdee, A Charles \& J Seijo. 2011. Coastal fisheries of Latin America and the Caribbean region: issues and trends. FAO Fisheries and Aquaculture Technical Paper 544: $1-430$

Sangronis C. 1991. Estructura etaria del camarón blanco Litopenaeus schmitti presente en la Ciénaga Los Olivitos, Zulia-Venezuela. Tesis de Licenciatura, Universidad del Zulia, Maracaibo, 84 pp.

Sparre P \& S Venema. 1997. Introducción a la evaluación de recursos pesqueros tropicales. Parte 1: Manual. FAO Documento Técnico de Pesca 306: 1-420.

Wetherall J. 1986. A new method for estimating growth and mortality parameters from length-frecuency data. Fishbyte 4: $12-14$.

Zar J. 1996. Biostatistical analysis, 662 pp. Prentice Hall, Englewood Cliffs. 